\title{
Experiencia de Escape Room de Fisiología
}

\section{Physiology Escape Room Experience}

DOI: $10.46932 /$ sfjdv2n5-044

Received in: Oct 1st, 2021

Accepted in: Dec 30th, 2021

\section{María-Isabel Jiménez-Serranía $\mathrm{PhD}$}

Institution: Department of Health Science. European University of Miguel de Cervantes (UEMC)

Address: C/Padre Julio Chevalier, 2, 47012. Valladolid (España).

C/ Padre Julio Chevalier, 2, 47012. Valladolid (SPAIN). Room 2230

Tel: 983001000 Ext.22301/ (+34)654781896

E-mail: ijimenez@uemc.es

\section{Noemí Yubero Postigo}

$\mathrm{PhD}$

Institution: Department of Health Science. European University of Miguel de Cervantes (UEMC) Address: C/Padre Julio Chevalier, 2, 47012. Valladolid (España).

E-mail: nyubero@uemc.es

\section{Sonia M. Gallego Sandín}

$\mathrm{PhD}$

Institution: Department of Health Science. European University of Miguel de Cervantes (UEMC) Address: C/Padre Julio Chevalier, 2, 47012. Valladolid (España).

E-mail: sgallego@uemc.es

\section{Inmaculada Fierro Lorenzo $\mathrm{PhD}$}

Institution: Department of Health Science. European University of Miguel de Cervantes (UEMC)

Address: C/Padre Julio Chevalier, 2, 47012. Valladolid (España).

E-mail: ifierro@uemc.es

\section{Eduardo Miguel Velado}

$\mathrm{PhD}$

Institution: Department of Health Science. European University of Miguel de Cervantes (UEMC)

Address: C/Padre Julio Chevalier, 2, 47012. Valladolid (España)

E-mail: emiguel@uemc.es

\section{Raquel Martínez Sinovas $\mathrm{PhD}$}

Institution: Department of Health Science. European University of Miguel de Cervantes (UEMC)

Address: C/Padre Julio Chevalier, 2, 47012. Valladolid (España).

E-mail: rmsinovas@uemc.es 


\section{RESUMEN}

Objetivo. El presente proyecto ha evaluado la aplicación de la actividad de gamificación Escape Room como técnica para aumentar la dinamización y participación activa del alumnado en su formación en Fisiología dentro de un entorno de trabajo cooperativo, así como su percepción y evaluación indirecta de los conocimientos teóricos mediante su aplicación en un aprendizaje basado en resolución de problemas. Material y método. Se ha planificado y aplicado un Escape Room en estudiantes de Grados en Ciencias de la Actividad Física y del Deporte (CAFD) y Terapia ocupacional (TO) que cursan la asignatura transversal de Fisiología ambientado en un laboratorio experimental. Se planificó una estrategia no lineal y competitiva enfrentando a dos grupos de alumnos en espacios separados. Se realizó una encuesta de satisfacción al alumnado al término de la prueba.

Resultados. El número total de participantes que realizó la actividad fue de 87. La implicación y el grado de satisfacción de los alumnos con la actividad fue muy elevado.

En términos generales, el 60,8\% del alumnado consideró esta actividad 'muy divertida' (5 puntos sobre 5), el 75,9\% tuvo sensaciones positivas al realizarla, el 86,1\% considera 'haber aprendido algo', el 87,3\% recomendaría a sus compañeros realizar esta actividad y al 87,3\% le gustaría repetir esta actividad con otras asignaturas o contenidos.

Conclusiones. La técnica de gamificación Escape Room en Fisiología ha demostrado aumentar el interés y motivación por la materia, poniendo de manifiesto puntos fuertes y débiles en su conocimiento aplicado y promoviendo la formación y cooperación en grupo.

Keywords: gamificación, sistemas fisiológicos, motivación.

\section{SUMMARY}

Objective. The present project has evaluated the application of the gamification activity Escape Room as a technique to increase the dynamization and active participation of students in their training in Physiology within a cooperative work environment, as well as their perception and indirect evaluation of theoretical knowledge through its application in a learning based on problem solving.

Material and Method. An Escape Room has been planned and applied in students of Physical Activity and Sport Sciences (CAFD) and Occupational Therapy (OT) taking the cross-cutting subject of Physiology set in an experimental laboratory. A non-linear and competitive strategy was planned, pitting two groups of students against each other in separate spaces. A student satisfaction survey was carried out at the end of the test.

Results. The total number of participants who took part in the activity was 87 . The involvement and degree of satisfaction of the students with the activity was very high.

In general terms, $60.8 \%$ of the students considered this activity 'very fun' (5 points out of 5), $75.9 \%$ had positive feelings when doing it, $86.1 \%$ considered 'having learned something', $87.3 \%$ would recommend this activity to their classmates and $87.3 \%$ would like to repeat this activity with other subjects or contents. Conclusions. The gamification technique Escape Room in Physiology has shown to increase interest and motivation for the subject, highlighting strengths and weaknesses in their applied knowledge and promoting group training and cooperation.

Keywords: gamification, physiological systems, motivation.

\section{INTRODUCCION}

Es un hecho evidente que algunas asignaturas básicas en la formación de cualquier profesional de Ciencias de la Salud resulten de bajo interés para el alumnado, bien por falta de conocimientos previos o 
bien por falta de visión de aplicación en su futura profesión. Sin embargo, los conocimientos de Fisiología actúan de base sólida para ejercer su futuro trabajo de forma profesional con conocimiento profundo del funcionamiento del organismo, desde el nivel de órganos y sistemas hasta el funcionamiento celular y molecular, por lo que dominar esta materia es clave para convertirse en un buen profesional [1,2].

A su vez, la participación activa y colaboración grupal en los primeros cursos está afectada por muchos factores entre los que destacan las capacidades de adaptación al nuevo entorno de aprendizaje y de interacción con nuevos compañeros que en ocasiones puede resultar difícil por falta de motivación para relacionarse $[3,4]$.

Esta situación nos impulsó a plantear una actividad de gamificación que consiguiese dinamizar los contenidos de una asignatura eminentemente teórica como es Fisiología, especialmente teniendo en cuenta que el juego se utiliza como base del aprendizaje por las sociedades desde hace siglos, adaptándolo actualmente a los de mesa, roll y a posteriori videojuegos [5]. En este trabajo se plantea promover la participación de los estudiantes y 'crear equipo' mediante el trabajo cooperativo y competitivo.

De entre las múltiples actividades de gamificación utilizadas en el ámbito académico, consideramos que la modalidad de Escape Room (también denominado Breakout Edu [6] en su ámbito de aplicación en la enseñanza) era la más adecuada. Esta estrategia permite aumentar la motivación intrínseca a través de la inmersión mental en una 'experiencia de flujo' satisfactoria, desarrollar competencias de aprendizaje basado en problemas permitiendo aplicación práctica de la teoría, así como estimular la participación y colaboración grupal [7,8]. Su aplicación como estrategia de enseñanza superior en Ciencias de la Salud ya ha demostrado resultados positivos [9].

En el ámbito universitario, también existen estudios en los que este tipo de técnicas demostraron reducir el estrés del alumnado hacia los contenidos, incrementaban la motivación y el compromiso con la asignatura, permitían la identificación de las habilidades de los estudiantes y mejoraban la adquisición de los conocimientos y competencias clave de las materias [10].

\section{OBJETIVOS}

El objetivo principal del presente proyecto ha sido diseñar y evaluar la aplicación de la actividad de gamificación Escape Room como técnica para aumentar la dinamización y participación activa de los alumnos en la formación en Fisiología dentro de un entorno de trabajo cooperativo, así como su percepción y evaluación indirecta de los conocimientos teóricos mediante su aplicación en un aprendizaje basado en resolución de problemas.

Los objetivos secundarios fueron:

- Valorizar la formación de aspectos clave en Fisiología para su posterior aplicación.

- Consolidar conocimientos sobre los que no se hubiese profundizado. 
- Localizar puntos débiles en la adquisición de conocimientos básicos.

- Detectar interacciones y potenciales mejoras en la relación alumno-alumno y docente-alumno.

\section{METODOLOGÍA}

\section{1 ÁMBITO DE APLICACIÓN}

Se ha planificado y aplicado un Escape Room de Fisiología en grupos de estudiantes de Grados en Ciencias de la Actividad Física y del Deporte (CAFD) y Terapia ocupacional (TO) que cursan la asignatura transversal de Fisiología en los siguientes grupos (ver Tabla 1).

Tabla 1. Descriptiva de los Grados en las que se ha aplicado Escape Room de Fisiología

\begin{tabular}{|c|c|c|c|}
\hline Grados / Grupo & Curso/tipo & Acrónimo & $\begin{array}{c}\text { Alumnos } \\
\text { matriculados }\end{array}$ \\
\hline $\begin{array}{c}\text { Ciencias de la actividad física y del deporte (CAFD) } \\
\text { / M1 }\end{array}$ & $1^{\circ} /$ básica & CAFD & 36 \\
\hline $\begin{array}{c}\text { Ciencias de la actividad física y del deporte (CAFD) } \\
\text { / M2 }\end{array}$ & $1^{\circ} /$ básica & CAFD & 39 \\
\hline Terapia Ocupacional (TO) & $1^{\circ} /$ básica & TO & 12 \\
\hline Total & & & 87 \\
\hline
\end{tabular}

\subsection{ESTRATEGIA}

Ambientación. El Escape Room implementado está inspirado en la película de Richard Fleischer 'Viaje alucinante' y posterior obra de Isaac Asimov ambas estrenada y escrita, respectivamente, en 1966 [11]. Narra la historia fantástica de un viaje al interior del cuerpo humano con un submarino tripulado que ha sido reducido de tamaño en el Centro de Miniaturización Norteamericano. Se preparó una ambientación sencilla al realizarse en aulas con clases previas y posteriores a la prueba, con audio de entradilla, imagen de fondo y cronómetro con música ambiente en pantalla.

Instrucciones. Se facilitaron instrucciones de realización a cada grupo al inicio de la actividad, para su correcta realización, recalcando la organización, la comunicación y la colaboración entre ellos.

Diagrama de flujo. Se planificó una estrategia no lineal y competitiva enfrentando a 2 equipos de cada grupo de alumnos en espacios separados [12].

El itinerario a través de los diferentes sistemas fisiológicos se realiza con el objetivo de probar los conocimientos del alumnado. Se pueden resolver varias pruebas a la vez y el orden de resolución puede variar según el momento en el que se encuentren las pistas con puntos de confluencia (Figura 1).

Pruebas. Se desarrolló una batería de pruebas para cada paso a superar, así como las "pistas" que se ofrecería a los alumnos para ayudarles a superar las diferentes pruebas con el objetivo de que alcanzaran el resultado final. 
Posteriormente, se realizó una selección consensuada de la batería de pruebas que mejor se adaptaran al contenido al considerar la transversalidad de la asignatura entre Grados de Ciencias de la Salud.

Cada prueba constaba de un problema asociado a un sistema fisiológico y un sistema de codificación basado en conceptos de Fisiología que darían la clave para abrir la siguiente prueba y avanzar hasta el punto final (Figura 1).

Se establecieron las siguientes confluencias a lo largo del flujo por el Escape Room para interrelacionar las pruebas de los diferentes sistemas:

- División celular - Sistema respiratorio

- Sistema nervioso - Sistema digestivo

- Sistema circulatorio - Sistema endocrino

El itinerario final contenía las siguientes pruebas de conceptos básicos relativos a todos los aparatos y sistemas fisiológicos:

- sopas de letras y puzzles de secuencias de imágenes para las pruebas en biología celular, sistema nervioso y sistema circulatorio

- términos con cuestionario e identificación en imagen para las pruebas en sistema respiratorio, sistema digestivo y sistema endocrino

- puzzle acertijo final colaborativo.

Tras resolver el puzzle cooperativo y abrir la caja final reciben una recompensa por subgrupo ('píldoras miniaturizantes') y un premio sorpresa al mejor tiempo entre subgrupos para incentivar la competición (bolsa serigrafiada UEMC).

Pruebas piloto. Se realizaron dos pruebas piloto seriadas para mejorar la fluidez y perfeccionar las pruebas. Se acuerda facilitar pistas, en especial en las pruebas piloto, para permitir el avance de la actividad.

Aplicaciones: Se aplica en 3 ocasiones. Las pruebas y tiempos se adaptan según el número de alumnos que realizan la prueba en cada grupo.

Evaluación. Los alumnos realizaron una valoración anónima mediante rúbrica vía Moodle (formato tipo test para facilitar su realización) con el móvil incluyendo el grado de satisfacción con la actividad realizada, percepción de aprendizaje y apreciaciones sobre el modelo colaborativo. Las preguntas de la encuesta están basadas en las estandarizadas para el análisis de una actividad de gamificación analizada previamente por los autores (en concreto la encuesta de satisfacción de la herramienta de gamificación Kahoot). 
Tras la experiencia, los docentes participantes en el proyecto también realizaron una evaluación del desarrollo del proyecto mediante rúbrica, con el fin de mejorarlo y adaptarlo de forma más efectiva a las necesidades del alumnado en ediciones futuras.

Figura 1. Diagrama de flujo de Escape Room en Fisiología.

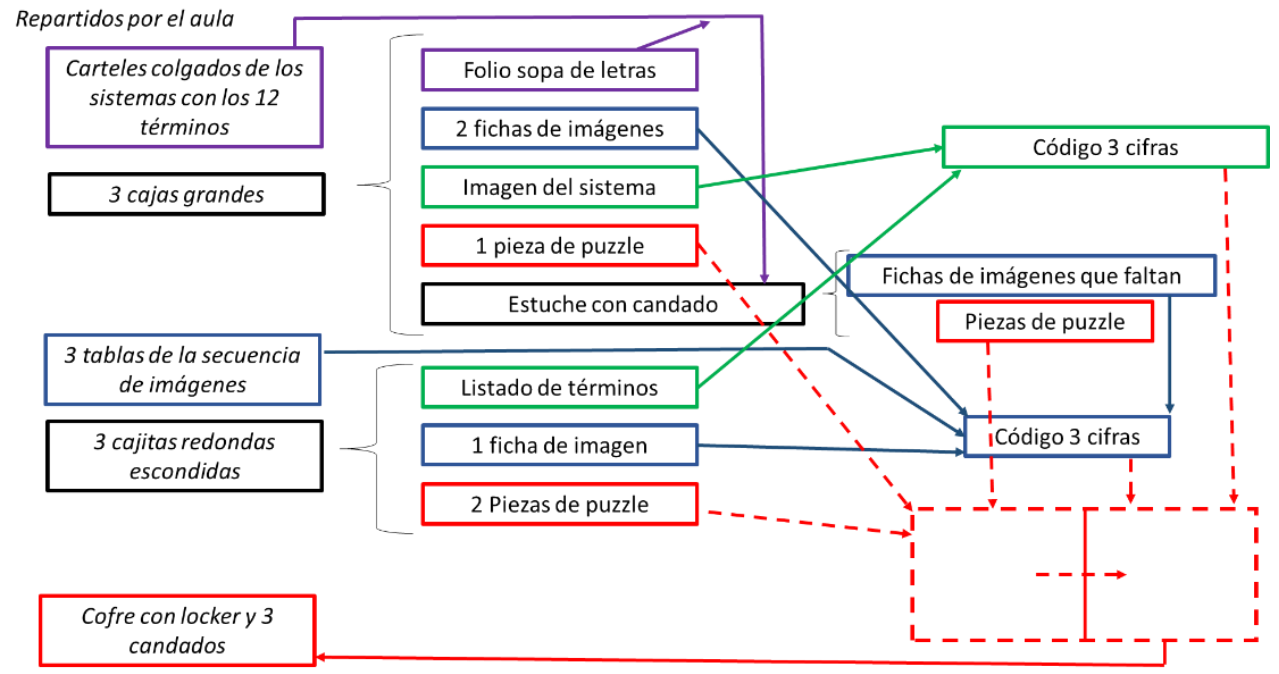

\section{RESULTADOS}

El número total de estudiantes a los que se aplicó el modelo final fue de 87. Se realizó en un Grado con un número elevado de alumnos matriculados (CAFD) y en un Grado con un número bajo de matriculados (TO) (Tabla 1).

En el análisis de los resultados hay que considerar dos apartados: resultados formativos y resultados de implementación.

\subsection{RESULTADOS FORMATIVOS}

- Se observa un aumento de interés por la asignatura tras realizarse una actividad más dinámica con un contenido eminentemente teórico.

- Los propios alumnos reconocen sus carencias formativas durante la realización y, posteriormente, hacen referencias constantes durante el cuatrimestre a aspectos abordados en las pruebas.

- Se detectan puntos fuertes y débiles en la formación básica en Ciencias de la Salud y su divergencia según el historial formativo de los alumnos. Los provenientes de Bachillerato de Ciencias de la Salud poseen conocimientos más sólidos y recientes que los que proceden de Bachillerato de Ciencias Sociales o de Formación Profesional (TAFAD, Técnico Superior en Animación de Actividades Físicas y Deportivas).

- Se detecta la necesidad de tener una referencia sobre los conocimientos previos y su experiencia en este tipo de actividades. 


\subsection{RESULTADOS DE IMPLEMENTACIÓN}

- La participación activa fue muy elevada en todos los grupos. Este resultado parece no verse afectado por el número de participantes que realiza la actividad.

- La creación de una comunicación directa con el profesor para resolver dudas rompe la barrera docente-alumno, generando una interacción más cercana y motivando al alumnado a resolver sus consultas.

- El hecho de ver un comportamiento de interés y participación por parte de los alumnos más aventajados anima al grupo a implicarse en la actividad y aumentar la comunicación alumno-alumno.

- La recompensa final y el espíritu competitivo son los objetivos para los que muestran más interés.

Se realiza un análisis normalizado de los resultados obtenidos en las pruebas de evaluación llevadas a cabo tanto a los docentes sobre el desarrollo del proyecto como a los alumnos sobre satisfacción con la actividad.

\subsection{EVALUACIÓN DE LOS DOCENTES}

Se realiza una evaluación conjunta de las percepciones ya que cada docente participó en implementaciones diferentes.

En términos generales, los logros conseguidos, la motivación del alumnado y la valoración global de la actividad fueron muy satisfactorios desde la prueba piloto 1.

La comprensión de las normas de la actividad fue el aspecto que mejoró más rápidamente, seguido de la adecuación de la actividad al tiempo previsto.

El aspecto que mejoró más paulatinamente fue la organización de la actividad, siendo la última implementación la más satisfactoria.

La impresión inicial al comenzar la actividad es que el alumnado se mostraba interesado en la tarea que debían realizar. Además, según se iba avanzando en la dinámica, se generaba una mayor implicación de todos los participantes en la misma, efecto que debe producirse en este tipo de metodología, lo que permite deducir que el diseño es correcto.

\subsection{ENCUESTA DE SATISFACCIÓN DE LOS ALUMNOS}

Conociendo la escasez de opiniones orales y objetivas que suele plantear una pregunta de opinión expuesta por el docente, consideramos relevante obtener más información de la percepción de la actividad a través del análisis de las respuestas de la encuesta final.

El porcentaje de alumnos matriculados por asignatura que realizan la encuesta es superior al $90 \%$. 
En términos generales, el 60,8\% del alumnado consideró esta actividad 'muy divertida' (5 puntos sobre 5), el 75,9\% tuvo sensaciones positivas al realizarla, el $86,1 \%$ considera 'haber aprendido algo', el $87,3 \%$ recomendaría a sus compañeros realizar esta actividad y al 87,3\% le gustaría repetir esta actividad con otras asignaturas o contenidos.

Analizando pormenorizadamente por Grado (Figuras 2-7), y ordenando de mayor a menor puntuación, las mejores valoraciones como 'muy divertida' serían para TO (90,0\%) y para CAFD $(56,5 \%)$; sensaciones positivas para TO $(80,0 \%)$ y CAFD $(75,4 \%)$; haber aprendido algo para TO $(100 \%)$ y CAFD (84,1\%); y recomendarían esta actividad para TO (100\%) y CAFD (85,5\%). Entre las respuestas de texto libre procesadas sobre el interés que les suscita la Fisiología las más frecuentes fueron Integración del conocimiento para TO (40\%) y Funcionamiento del cuerpo para CAFD $(34,8 \%)$.

Todas las preguntas presentan más respuestas positivas que neutras o negativas. Este patrón se repite en los dos Grados analizados.

En TO todos los estudiantes contestan a todas las preguntas. En CAFD existe un porcentaje bajo de alumnos que no contesta alguna pregunta de respuesta fija (14,5\%; 9 alumnos acceden a la encuesta, pero no contestan ninguna pregunta); el porcentaje aumenta en la respuesta de texto libre (30\%).

\subsection{LIMITACIONES DEL ANÁLISIS}

Dado que la actividad se realiza al inicio del cuatrimestre, no se puede valorar como trabajo en grupo para las calificaciones ni el resultado (el nivel hasta el que se ha llegado) ni el tiempo de resolución de cada prueba, al no partir todos los alumnos de la misma base formativa. Por idéntico motivo, no es posible realizar un análisis de evolución pre-post de los resultados de la actividad.

\subsection{MEJORAS A REALIZAR PARA FUTURAS IMPLEMENTACIONES}

Mejorar la ambientación con elementos físicos (p. ej. realizarla en uno de los laboratorios experimentales) y hacer una valoración simple de conocimientos iniciales para adaptar mejor las pruebas.

Figura 2. Respuestas por Grado a la pregunta 1 de la encuesta: Valora de 1 (nada divertida) a 5 (muy divertida) esta actividad.

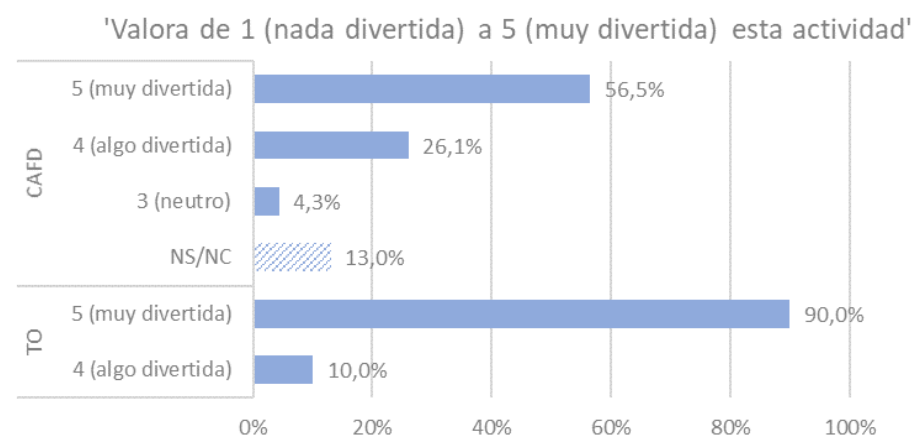


Figura 3. Respuestas por Grado a la pregunta 2 de la encuesta: ¿Cómo te has sentido tras realizarla?

'Cómo te has sentido al realizarla'

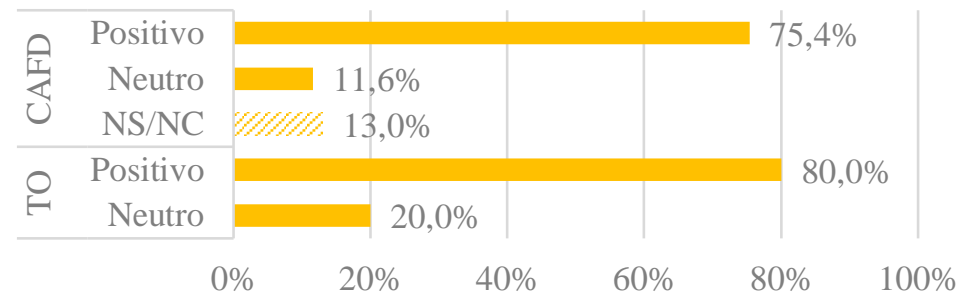

Figura 4. Respuestas por Grado a la pregunta 3 de la encuesta: Indica verdadero/falso si 'has aprendido algo'.

'Has aprendido algo'

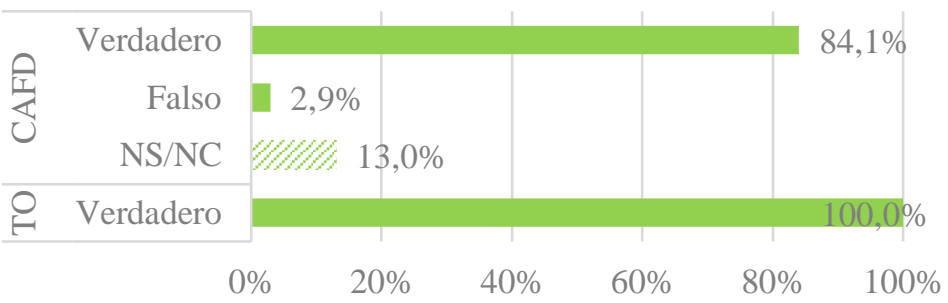

Figura 5. Respuestas por Grado a la pregunta 4 de la encuesta: Indica verdadero/falso si 'recomendarías realizar esta actividad a tus compañeros'

\section{'Recomendarías realizar esta actividad a tus compañeros'}

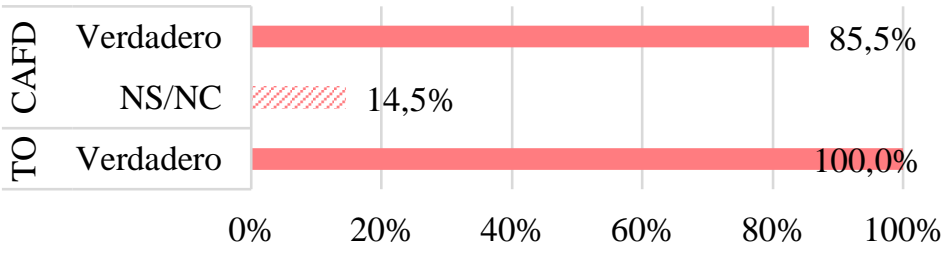

Figura 6. Respuestas por Grado a la pregunta 5 de la encuesta: Indica verdadero/falso si 'te gustaría repetir esta actividad con otras asignaturas o contenidos'.

'Te gustaría repetir esta actividad con otras asignaturas o contenidos'

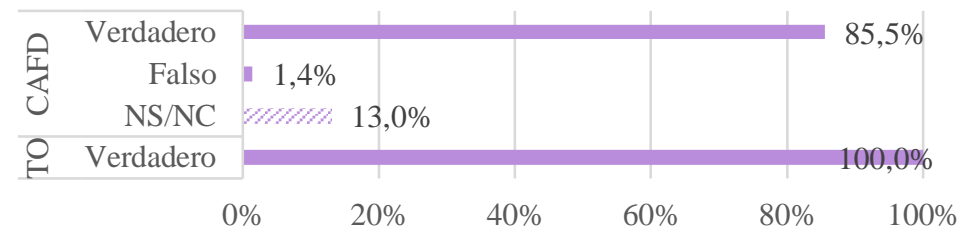


Figura 7. Respuestas por Grado a la pregunta 6 de la encuesta: 'Después de realizar esta actividad, ¿qué es lo que más te llama la atención de la Fisiología? ¿y lo que menos?' (respuesta libre; procesada).

'Después de realizar esta actividad, ¿qué es lo

que más te llama la atención de la

Fisiología? ¿y lo que menos?' (respuesta libre;

procesada)

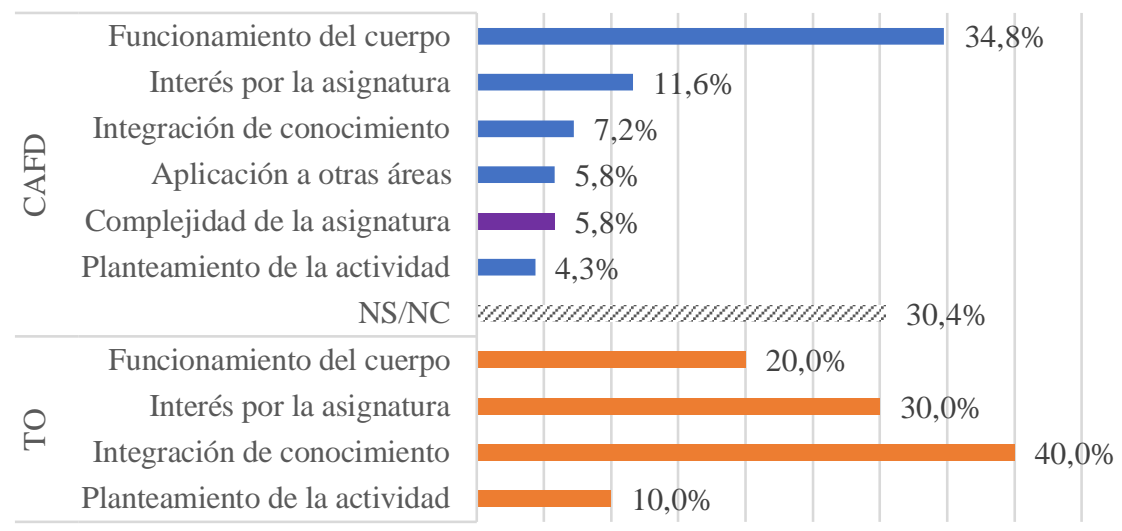

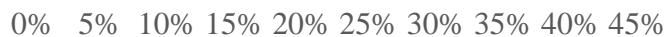

\section{CONCLUSIONES}

La técnica de gamificación Escape Room en Fisiología ha demostrado cambiar la percepción del alumnado por la asignatura de Fisiología, aumentando su interés y motivación hacia la misma durante el cuatrimestre.

El descubrimiento de las carencias formativas globales sirve de referencia para profundizar en ellas, consolidar conocimientos y generar una base común desde la que avanzar a conceptos más complejos.

La promoción del trabajo cooperativo pone de manifiesto la necesidad de desarrollar competencias grupales y genéricas -trabajo en equipo, toma de decisiones, habilidades interpersonales- entre los universitarios.

\section{FINANCIACIÓN}

Este estudio se realizó en el marco del Proyecto de Innovación Educativa PIE13-1920 'Escape Room de Fisiología' y ha sido financiado por la Convocatoria de Proyectos de Innovación Educativa 2019-2020 de la Universidad Europea Miguel de Cervantes (UEMC).

\section{AGRADECIMIENTOS}

A la Universidad Europea Miguel de Cervantes (UEMC) por permitir y financiar la realización del Proyecto de Innovación Educativa PIE13-1920 'Escape Room de Fisiología'. 


\section{REFERENCIAS}

[1] Bowen JL. "Educational Strategies to promote clinical diagnostic reasoning". N Engl J Med. 355(21):2217-25. doi: 10.1056/NEJMra054782 (2006).

[2] Castañeda MT, Rodríguez HE, Castillo O, Daniel E, Rodríguez JM. "El razonamiento clínico desde el ciclo básico, una opción de integración en las ciencias médicas”. Edumecentro, 7(1):18-30 (2015).

[3] Delors J. "Education: the necessary utopia. Learning: The Treasure Within". Report of the International Commission on Education for the Twenty-first Century. Paris: UNESCO (1996).

[4] Monedero C, Durán D. "Entramados: métodos de aprendizaje cooperativo y colaborativo". Madrid: Édebe (2002).

[5] Burke B. "Innovation Insight: Gamification Adds Fun and Innovation to Inspire Engagement". Gartner Research, 2011, https://www.gartner.com/en/documents/1879916 (10 de septiembre de 2020).

[6] Breakout, Inc., dba Breakout EDU, 2018, www.breakoutedu.com (11 de septiembre de 2020).

[7] Jambhekar K, Pahls RP, Deloney LA. Benefits of an Escape Room as a Novel Educational Activity for Radiology Residents. Acad Radiol. 27(2):276-283. doi: 10.1016/j.acra (2020).

[8] Csikszentmihalyi M. "Fluir: una psicología de la felicidad”. Editorial Kairós, Barcelona (2010).

[9] Mawhirter DA, Garofalo PF. "Expect the Unexpected: Simulation Games as a Teaching Strategy". Clinical Simulation in Nursing 12(4), 132-136 (2016).

[10] Alsawaier R. "The effect of gamification on motivation and engagement". The International Journal of Information and Learning Technology 35:56-79. (2017).

[11] Asimov I. Viaje alucinante. Bantam Books, Nueva York (1966).

[12] Instituto de la Juventud de Extremadura. "Manual de Diseño de un Juego de Escape". Junta de Extremadura, 2018, http://culturaemprendedora.extremaduraempresarial.es/wpcontent/uploads/2018/04/Manual-de-Escape.pdf. (14 de junio de 2019). 